\title{
Zur Kenntniss der Elementarzusammensetzung der Reisstärke und der quantitativen Bestimmung derselben;
}

von

\section{F. Salomon. $\left.{ }^{1}\right)$}

In meiner kürzlich ${ }^{2}$ ) veröffentlichten Arbeit über: „Die Elementarzusammensetzung der Stärke" habe ich S. 354 darauf aufmerksam gemacht, dass möglicherweise die von verschiedenen Pflanzen stammende Stärkearten eine verschiedene chemische Zusammensetzung besitzen könnten.

Um diese Ansicht zu prüfen, hatte ich schon vor längerer Zeit Herrn stnd. Schulze veranlasst, die Weizenstärke eingehend zu untersuchen, während ich selbst die Reisstärke in Angriff genommen habe.

Die Resultate meiner Untersuchungen gebe ich im Folgenden, diejenigen des Herrn Schulze sind gleichfalls abgeschlossen und werden in Kurzem zur Veröffentlichung gelangen.

Zur Aufnahme dieser Arbeit bin ich wesentlich mit veranlasst, durch private Mittheilungen des Herm Felix Allihn, wonach die Reisstärke bei der Verzuckerung mit 0,3 bis 0,5 proc. verdünnter Schwefelsiture unter Druck und nachleriger Bestimmung der entstandenen Zuckermenge ${ }^{3}$ ) nach Allihn's Methode höchstens bis zu 95 Procent verzuckert wird, eine Beobachtung, welche, wie aus den folgenden Zeilen zu ersehen ist, durch meine Versuche bestätigt ist.

Der für den vorliegenden Zweck einzuschlagende Weg war mir schon durch meine eiste Arbeit über die Kartoffelstärke nahegelegt. Wenn der Elementarbestand der Reis-

1) Mittheilung aus dem technisch-chemis hen Laboratorim der Herzogl. techn. Hochschule zu Braunschweig.

2) Jies. Journ. [2] 25, 348 .

3) Nach dicsem Verfahren wurden sciner 'Leit in der Hoffmann'schen Reisstärkefabrik zu salzutfeln Stäkeanalysen ausgeführt. 
der Reisstärke u. der quantit. Bestimmg. derselben. 325

stärke von der Formel $\left(\mathrm{C}_{6} \mathrm{H}_{10} \mathrm{O}_{5}\right)_{\mathrm{a}}$ wesentlich abwich, so musste auch die Menge der durch Behandlung mit verdünnten Mineralsäuren entstehenden Umwandlungsprodukte von 111,1 Procent wesentlich verschieden sein. Bei Gelegenheit meiner Untersuchung über das spec. Gewicht und das optische Verhalten des Traubenzuckers (Dextrose) ${ }^{1}$ ) hatte ich mich schon durch eingehende Versuche überzeugt, dass der aus der Reisstärke gebildete Zucker in physikalischen wie chemischen Eigenschaften mit dem aus der Kartoffelstärke gebildeten Zucker identisch ist, somit war es hinreichend, die Quantität der Endprodukte, welche bei der Hydrolyse der Reisstärke entstanden, allein zu berücksichtigen.

Die näheren Details der Ausführung dieser Versuche ergaben sich am besten aus der im Eingange angeführten Abhandlung; ich habe, um unliebsame Wiederholungen zu vermeiden, die Darstellung der Versuchsresultate thunlichst verkürzt.

\section{Versuchsreihe I.}

$\mathrm{Zu}$ diesen Versuchen fand ein dem Anschein nach sehr reines Fabrikat Verwendung, welches von der Firma G. Lange in Bremen als feinste Reis-Strahlenstärke in den Handel kommt.

Die technische und mikroskopische Prüfung erwiesen die Waare als vollständig tadellos und frei von anderen Stärkearten.

Was die Ausführung der Analysen dieser, sowie der zu Versuchsreihe II benutzten Stärke anlangt, so sind beide nach der Methode vorgenommen, welche ich seiner Zeit im Repertorium für analyt. Chemie 1, 274 näher beschrieben habe. Die Untersuchung der vorliegenden Handelswaare gab folgende Werthe:

a) Wasergehalt:

1) 4,180 Grm. lufttr. Stärke hinterliessen nach dem Trocknen bei $120^{\circ}=3,519$ Grm. wasserfr. Substanz $=15,82 \%$ Wasser.

2) $3,802 \mathrm{Grm}$. lufttr. Stärke, bei $120^{\circ}$ getrocknet, lieferten 3,197 Grm. Wasserfr. Subst. $=15,91 \%$ Wasser.

1) Siehe Repert. f. anal. Chem. 1, 309. 
326 Salomon: Zur Kenntu. d. Elementarzusammensetz.

3) 5,663 Grm. lufttr. Stärke, bei $120^{\circ}$ getrocknet, lieferteu 4,767 Grm. wasserfr. Subst. $=15,82 \%$ Wasser.

Daraus in Mitel:

$$
\begin{aligned}
& \text { \$4,15 Grm. Trockensubstanz } \\
& 15,85 \quad " \quad \text { Wasser }
\end{aligned}
$$

in 100 Thln. Iufttrockner Substanz.

b) In kochenden verdünnten Säuren unlösliche: Rückstand:

1) 4,296 Grm. lufttr. Subst. gaben 0,032 Grm. unlösl. Ritckstand $=0,77 \%$.

2) 3,5565 Grm. lufttr. Subst. gaben 0,0225 Grm. unlösl. Rück$\operatorname{stand}=0,63 \%$.

3) 4,131 Grın. lufttr. Subst. gaben 0,025 Grm. unlösl. Rückstand $=0,71 \%$.

Daraus in Mittel:

0,703 pCt. unlöslicher Rückstand.

c) Aschenbestimmung:

1) $7,688 \mathrm{Grm}$. Stärke gaben $0,0525 \mathrm{Grm}$. Asche $=0,628 \%$.

2) Eine früher ausgefühte Bestimnung ergab $=0,607 \%$.

Daraus in Mittel:

$$
0,618 \text { pCt. Asche. }
$$

Nach diesen Analysen enthält die lufttrockne Reisstärke I folgende Componenten:

$\begin{array}{llc}\text { Reine Stärke } & = & 82,84 \\ \text { Asche } & = & 0,607 \\ \text { Unlöslicher Rückstand } & = & 0,703 \\ \text { Wasser } & = & 15,850 \\ & & 100,000\end{array}$

Verzuckerungsversuche mit Reisstärke I (Handelswaare) nach Sachsse's Methode und Bestimmung des Zuckers nach Allihn's Vorschrift. $\left.{ }^{1}\right)$

$$
\text { Versuch I. }
$$

$4,296 \mathrm{Grm}$. lufttr. Stärke $=3,559 \mathrm{Grm}$. reiner wasserfr. Stärke in einen Kolben gespült und mit $200 \mathrm{Ccm}$. Wasser und $20 \mathrm{Cem}$. veiner Salzsäure (spec. Gew. 1,125) versetzt. Die Flüssiglseit nach 3stündigem Erhitzen im Wasserbade mit Kalilauge annähernd neutralisirt und auf 500 Cem. verdünnt.

1) Ueber diese Methode: dies. Journ. [2] 95, 354, und Repert. f. anal. Chem. 1, 274, 309. 
der Reisstärke u. der quantit. Bestimmg. derselben. 327

$25 \mathrm{Cem}$. der so entstandenen Zuckerlösing lieferten $361 \mathrm{Mgrm}$. $\mathrm{Cu}$, cntsprechend 190,6 Mgm. Zucker, also in 500 Ccm. $=20 \times 190,6$

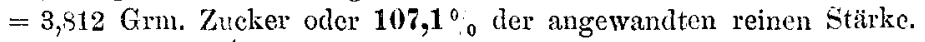

\section{Versuch II.}

3,5565 Grm. lufttr. Stärke $=2,946$ Gr'n. reiner wasserfi. Subst. gaben im Mittel für $25 \mathrm{Ccm}$. der verdünnten Lösung 303,5 Mgrm. Cu, äquivalent 158,5 $\mathrm{Mgrm}$. Zucker, entsprech. 3,170 Grm. Dextrose oder $107,5 \%$ der verbrauchten Stärke.

\section{Versuch III.}

$4,131 \mathrm{Grm}$. Iufttr. Stärke $=3,422 \mathrm{Grm}$. reiner wasserfs. Substanz gaben in Mittel für $25 \mathrm{Ccm}$. der verdünnten Lösung $350 \mathrm{Mgrn}$. Cu, äquivalent 184,3 Mgrm. Zucker, entspr. 3,686 Grm. Dextrose oder $107,7^{\circ}$ o der angewandten reinen Stärke.

\section{Versuch IV.}

$3,377 \mathrm{Grm}$. lufttr. Stärke $=2,7975 \mathrm{Grm}$. reiner wasserfr. Stärke gaben im Mittel für $25 \mathrm{Ccm}$. der verdünnten Lösung $286 \mathrm{Mgrm}$. Cu = 148,8 Mgrm. Zucker, entspr. 2,976 Grm. Dextrose oder $\mathbf{1 0 6 , 4} \%$ der angewandten Stärke.

$$
\text { Versuch } V \text {. }
$$

$3,022 \mathrm{Grm}$. lufttr. Stäke $=2,503 \mathrm{Grm}$. reiner wasserfr. Substanz gaben im Mittel $260 \mathrm{Mgrm}$. Cu $=134,6 \mathrm{Mgrm}$. Zucker, entspr. 2,692 Grm. Dextrose oder

$\mathbf{1 0 7 , 1 5 \%} \%$ der angewandten Stärke.

Das Mittel aus diesen fünf Versuchen beträgt $107,17^{\circ} /$, d. h.: Bei der Verzuckerung der hier verbrauchten Reisstärke nach der Methode von Sachsse wurde eine vollständige Umwandlung in Dextrose nicht erreicht, da statt der zu erwartenden 111,1\% Dextrose nur 107,17\% erhalten werden konnten.

\section{Versuchsreihe II.}

Die in der vorhergehenden Versuchsreihe mit der käuflichen Reisstärke erzielten Resultate legten den Gedanken nahe, dass möglicherweise eine nicht genügend erkennbare Verunreinigung die Veranlassung der zu niedrigen Werthe für die Verzuckerung gewesen sei, und diese Vermuthung erschien um so wahrscheinlicher, als die fragliche Stärke 
328 Salomon: Zur Kenntn. d. Elementarzusammensetz.

ziemlich bedeutende alkalische Reaction zeigte, welche letztere bekanntlich auf die technische Gewinnungsmethode dieser Substanz aus dem Reis zurückzuführen ist.

Um den so vielleicht veranlassten Fehler möglichst auszuschliessen, habe ich eine grössere Partie derselben Bezugsquelle entnommener Reisstärke einer sorgfältigen Reinigung durch successives Behandeln mit äusserst verdünnter Salzsäure, Alkohol und Aether unterworfen, die dann vorsichtig an der Luft getrocknete und hierauf in gut verschliessbare Flaschen gebrachte Substanz analysirt und für die folgenden Versuche benutzt.

Die Resultate der Analyse waren folgende:

a) Wassergehalt:

1) 5,227 Grm. lufttr. gereinigter Reisstärke gaben bei $120^{\circ} 4,304$ Grm. 'Trockensubstanz

$$
=17,659 \% \text { Wasser. }
$$

2) 5,5485 Grm. lufttr. gereinigter Reisstärke licferten bei $120^{\circ}$ $4,5765 \mathrm{Grm}$. Trockensubstanz

$$
=17,518 \% \text { Wasser. }
$$

3) 4,2620 Grm. Iufttr. gereinigter Reisstärke lieferten bei $120^{\circ}$ 3,5085 Grm. Trockcnsubstanz

Daraus borechnet in Mittel:

$$
=17,680 \% \text { Wasser. }
$$

$82,381{ }^{\circ} i_{0}$ Trockensubst. und $17,619 \%$ Wasser.

b) Aschebestimmung:

1) 10,333 Grm. lufttr. Subst. gaben 0,0035 Grm. Asche

$$
=0,034^{\circ} \% \text { Asche. }
$$

2) 10,864 Grm. lufttr. Subst. gabon 0,0040 Grm. Asche

$$
=0,036 \%_{0}^{\circ} \text { Asche. }
$$

$$
\text { Mittel }=0,035 \% \text { Asehe. }
$$

c) Bestimmung des in verdïnnten Säuren unlösliclen Rückstandes:

20,93 Grm. gaben 0,021 Grm. unlöslichen Rückstand

$$
=0,1 \% \text { unlösl. Rüickstand. }
$$

Es ergiebt sich somit die Zusammensetzung der gereinigten Stärke:

$$
\begin{array}{llr}
\text { Reine Stärke } & =82,246 \\
\text { Asche } & =0,035 \\
\text { Unlösl. Rückstand } & =0,100 \\
\text { Wasser } & =17,619 \\
& & 100,000
\end{array}
$$


der Reisstärke u. der quantit. Bestimmg. derselben. 329

Verzuckerungsversuche mit der gereinigten Reisstärke nach der Methode von Sachsse und Bestimmung des Zuckers nach Allihn's Verfahren.

\section{Versuch VI.}

3,007 Grm. lufttr. Stärke $=2,473 \mathrm{Grm}$. reiner wasserfr. Substanz gaben im Mittel für $25 \mathrm{Ccm}$. der verdünnten Lösung $255 \mathrm{Mgrm}$. Cu = 131,9 Mgrm. Zucker, eutsprech. 2,633 Grm. Dextrose oder $106,7 \%$.

\section{Versuch VII.}

3,090 Grm. lufttr. Stïrke $=2,5415 \mathrm{Grm}$. reiner wasserfr. Subst. gaben im Mittel für $25 \mathrm{Ccm}$. der verdünnten Lösung $262 \mathrm{Mgrm}$. Cu $=135,7$ Mgrm. Zucker, entsprechend 2,714 Grm. Dextrose oder $\mathbf{1 0 6 , 8 \%}$.

\section{Versuch VIII.}

3,1305 lufttr. Stärke $=2,575$ Grm, reiner wasserfr. Subst, gaben im Mittel für $25 \mathrm{Ccm}$. der verdünnten Lösung 267,5 Mgrm. Cu = 138,7 Mgrm. Zucker, entsprechend 2,774 Grm. Dextrose

oder $107,7 \%$.

\section{Versuch IX.}

3,4425 Grm. lufttr. Stärke $=2,8315 \mathrm{Grm}$, reiner wasserfr. Subst. gaben im Mittel für $25 \mathrm{Ccm}$. verdünnter Lösung $289 \mathrm{Mgrm} . \mathrm{Cu}=150,5$ Mgrm. Zucker, entsprechend 3,010 Grm. Dextrose

$$
\begin{aligned}
& \text { oder 106,3 } \% \text {. } \\
& \text { Versuch } \mathrm{X} \text {. }
\end{aligned}
$$

$3,1835 \mathrm{Gr}$. lufttr. Stärke $=2,6184 \mathrm{Grm}$. reiner wasserfr. Subst. gaben im Mittel für $35 \mathrm{Ccm}$. der verdünnten Lösung $369 \mathrm{Mg} \mathrm{m}$. Cu $=195,1 \mathrm{Mgrm}$. Zucker, entsprechend 2,787 Grm. Dextrose in $560 \mathrm{Ccm}$. oder 106,4\%.

\section{Versuch XI.}

$4,3975 \mathrm{Grm}$. lufttr. Stärke $=3,617 \mathrm{Grm}$. reiner wasserfr. Substanz gaben im Mittcl für $25 \mathrm{Ccm}$. der auf $500 \mathrm{Ccm}$. verdünnten Lösung 365,5 Mgrm. Cu = 193,1 Mgrm. Zucker, entspr. 3,864 Grm. Dextrose oder 106,8\%.

Das Mittel aus diesen sechs Versuchen beträgt $106,8 \%$ somit ein Werth, welcher sehr gut dem durch die fünf Analysen der ersten Versuchsreihe festgestellten $(107,17 \%)$ correspondirt. Es muss hier gleich bemerkt werden, dass ich bei 
diesen letztgenannten Versuchen absichtlich zuweilen von der Vorschrift Sachsse's abwich. So wurde z. B. bei Versuch VII, VIII und IX die Kochdaner um eine Stunde vermehrt, bei Versuch XI auch die Menge der Salzsäure um $1 / 5$ erhöht; trotzdem ist, wie leicht au ersehen, keine merkbare Vermehrung in der Zuckerbildung eingetreten, und die Umwandlung sicher, soweit sie überhaupt möglich war, verlaufen. Auch der Einwurf, dass die Einwirkung der Säure auf die gebildeten Produkte zul weit gegangen sein könne, ist nicht stichhaltig, da sümmtliche Proben auch nach vollendeter Kochung vollständig farblos ersehienen.

Der zwingende Schluss, welcher das Resultat dieser elf Versuche darstellt, ist also folgender:

Bei den beiden vorliegenden Reisstärkesorten ist eine vollständige Ueberführ'ung in Traubenzucker nach Sachsse's Methode nicht zu erreichen; die analytisch nachweisbare Zuckermenge (Mittel 106,95) bleibt erheblich hinter der theoretisch berechneten und bei Kartoffelstärke seiner Zeit erzielten Quantität $111,11 \%$ zurück.

Hiernach schien es sehr naheliegend, anzunehmen, dass die Elementarzusammensetzung der Reisstärke von derjenigen der Kartoffelstärke abwiche, und es ist in der That nichts leichter als eine Formel aufzufinden, welche bei geringer Abweichung in der procentischen Zusammensetzung fast genau die durch meine Versuche gefundene Zuckermenge ergiebt.

Diese Formel ist $\mathrm{C}_{18} \mathrm{H}_{32} \mathrm{O}_{16}$ oder ein Multiplum davon, die Menge Zucker, welche aus einer derartig zusammengesetzten Stärke entstehen kann, ergiebt sich nach der Gleichung:

$$
\mathrm{C}_{18} \mathrm{H}_{32} \mathrm{O}_{16}+2 \mathrm{H}_{2} \mathrm{O}=3 \mathrm{C}_{6} \mathrm{H}_{12} \mathrm{O}_{6}
$$

zu 107,15 Proc., ist also fast absolut in der Versuchsreihe I ermittelt.

Die Abweichung in der Elementarzusammensetzung würde für $\mathrm{C} 1,6 \%$, für $\mathrm{H}$ nur $0,176 \%$ betragen und nach dem a. a. $\left.0 .{ }^{1}\right)$ Gesagten ist eine solche Differenz gerade bei der Stärke sehr schwierig durch die Elementaranalyse genau festzustellen. Die wunderbare Uebereinstimmung der analytisch gefundenen Werthe mit den berechneten hat gewiss viel Verlockendes, und ich muss in der That gestehen, dass ich eine Zeit lang fest daran glaubte, eine Verschiedenheit in der Zusammensetzung der beiden genannten Stärkearten gefunden zu haben, bis mich die späteren Versuche eines Besseren belehrten.

1) Dies. Journ. [2] 25, 348 u. f. 
der Reisstärke u. der quantit. Bestimmg. derselben. 331

Von früheren Erfallrungen geleitet, beschloss ich die genaue Bestimmung der durch die Behandlung mit verdünnten Säuren entstandenen Umwandlungsprodulte der Reisstärke durch die Feststellung des specifischen Gewichts. Bevor ich die weitere Discussion fortsetze, will ich mir erlauben, die hierbei erhaltenen Versuchsdaten anzugeben.

Feststellung der durch Verzuckerung der Reisstärke erhaltenen Umwandlungsprodukte mit Hülfe des specifischen Gewichts.

\section{Versuchsreihe III.}

Versuche mit der für Versuchsreihe I benutzten käuflichen Reisstärke.

Ans der oben angegebenen Zusammensetzung dieser Stïrke berechnet sich, dass, um je $100 \mathrm{Grm}$. der reinen absolut wasserfreien Stärke in den Versuch einzuführen, die Anwendung von 120,71 Grm. der lufttrocknen Waare erforderlich ist. Wie in der früheren, mit Kartoffelstärke ausgeführten Untersuchung (a. a. O. S. 357) brachte ich nur die Hälfte oder ein Viertel dieser Quantität in Anwendung, immer aber so viel, dass stets 10 Proc. der angewandten Stärkemenge in Lösung übergeführt werden mussten.

$\mathrm{Da}$ ich seiner Zeit, wie schon bemerkt, ausführlich über das Nähere derartiger Analysen berichtet habe, so wird es genügen, in aller Kürze das Nöthigste anzuführen:

\section{Versuch XII.}

A. $60,36 \mathrm{Grm}$. der lufttr. Stärke mit 0,9 proc. Schwefelsäure sechs Stunden im Kochsalzbade erhitzt, dann auf 500 Ccm. gebracht. ${ }^{1}$ )

Spec. Gewicht 1,0427 .

B. $250 \mathrm{Ccm}$. der Flüssigkeit von Versnch A, noch drei Stunden weiter gekocht.

Spec. Gewicht $=1,0426$.

Versuch XIII.

A. 60,36 Grma. der lufttr. Stärke mit 0,9 proc. Schwefelsäure 7,5 Stdn. gekocht, dann auf 500 Cem. verdünnt.

Spec. Gewicht $\doteq 1,0427$.

B. Nach weiterem Zusatz von 0,25 Proc. Schwefelsäure noch $11 / 2$ Stdn. gelkocht.

Spec. Gewicht $=1,042$.

Versuch XIV.

60,36 Grm. mit 0,9 proc. Schwefelsäure 12 Stdn. gekocht.

Spec. Gewicht $=1,04263$.

1) Das spec. Gewicht der $\mathrm{H}_{2} \mathrm{SO}_{4}$ vurde ganz wie in den früheren Versuchen bercchnet und abgezogen. 
332 Salom on: Kenntn. d. Elementarzusammensetz. etc.

Versuche mit der zur Versuchsreihe II benutzten gereinigten Reisstärke.

Für diese Stärke berechnet sich die Menge der für $100 \mathrm{Grm}$. absolut trockner und reiner Substanz anzuwendenden Stärke auf 121,6 Grm.

\section{Versuch XV.}

30,4 Grm. lufttr. Stärke, entsprechend $25 \mathrm{Gr}$. absolut trockner reiner Substanz, mit 1proc. Schwefelsäure 5 Stdn. gekocht, die Flüssigkeit auf $250 \mathrm{Ccm}$. gebracht.

Spec. Gewicht $=1,04223$.

\section{Versuch XVI.}

$30,4 \mathrm{Grm}$. lnfttr. Stärke, entsprechend $25 \mathrm{Grm}$. trockner reiner Substanz, mit 1proc. Schwefelsäure $6 \mathrm{Stdn}$. gekocht, dann die Flüssigkeit auf $250 \mathrm{Ccm}$. gebracht.

$$
\text { Spec, Gewicht = 1,04233. }
$$

Nimmt man wieder den Durchschnitt aus diesen verschiedenen Versuchen, so erhält man für die aus 10 Proc. Reisstärke durch Umwandlung in lösliche Produkte festgestellte Lösung ein mittleres spec. Gewicht von

$$
1,0425 \text {. }
$$

Vergleicht man diese Zahl mit dem Bd. 25, S. 361 unter ganz gleichen Bedingungen für aus 10 Proc. reiner Kartoffelstärke erhaltenen Werthe 1,0424, so erhellt, dass die Menge der beim Kochen von Reisstärke mit verdünnten Säuren entstehenden Produkte genau so gross ist, als die Quantität der unter gleichen Umständen aus der Kartoffelstärke gewonnenen Körper, nämlich 111,11 Proc. Dieselbe Procentzahl wurde bei Versuch XVI aus dem optischen Verhalten der gewonnenen Zuckerlösung abgeleitet. Die ermittelte Ablenkung betrug $34,0^{\circ}$ in Soleil-Ventzke-Scheibler'schen Apparate, somit war $\alpha j=58,76$ und der berechnete Gehalt an Dextrose 111,12\%

Dieses Resultat steht im scheinbaren Widerspruch mit den aus der Bestimmung des Traubenzuckers nach der combinirten Sachsse-Allihn'schen Methode ermittelten Daten, denm nur die Kartoffelstärke lieferte auch hier den für die Formel $\mathrm{C}_{6} \mathrm{H}_{10} \mathrm{O}_{5}$ allein zulässigen Werth von 111,11 Proc. Dextrose, während die Reisstärke, wie oben angeführt ist, nur 106,95 Proc. ergab. Es müssen also die fehlenden 4,16 Procent bei letzterer in andere, nicht reducirende Körper verwandelt sein.

\section{Schlussbemerknngen.}

Die vorliegende Arbeit liefert den Berweis, dass man nicht berechtigt ist, die mit Hülfe der Sachsse'schen Ver- 
Nencki u. Sieber: Ueber das Uroroseïn etc. 333

zuckerungsmethode, combinirt mit Allihn's Reductionsverfahren, gewonnenen analytischen Daten auf alle Stärkearten ohne Weiteres auszudehnen; es scheint vielmehr die Controle durch die Feststellung des specifischen Gewichtes der entstandenen Produkte unerlässlich. Die Constitution der Reisstärke, sowie ihre Elementarzusammensetzung ist höchst wahrscheinlich mit derjenigen der Kartoffelstärke identisch. Die niedrigen Zahlen, welche ich bei den Verzuckerungsversuchen erhielt, werden sich, wie ich glaube, auf eine Veränderung der Reisstärke bei ihrer technischen Gewinnung zurückführen lassen. Die Trennung dieser Stärke von den Zellmembranen erfolgt bekanntlich mit Hülfe verdünnter Laugen, und scheint es mir wahrscheinlich, dass bei diesem Vorgange ein, wenn auch geringer Procentsatz der Stärke derartig verändert wird, dass eine vollständige Umwandlung in Zucker nicht mehr erzielt werden kann, wohl aber die sämmtlichen Stoffe noch durch Kochen mit verdünnter Säure in Lösung gebracht werden und somit durch das specifische Gewicht zu ermitteln sind.

Immerhin würde es von Werth sein, durch weitere Versuche die Richtigkeit dieser Ansicht an Reisstärken verschiedener Abstammung zu prifen, und möchte ich interessirte Kreise bitten, diesen Gegenstand einer weiteren Prüfung zu unterwerfen.

Braunschweig, im October 1882.

\section{Teber das Uroroseïn, einen neuen Harnfarbstoff; von}

\section{Nencki und N. Sieber.}

Gelegentlich unserer Untersuchungen „über die physiologische Oxydation" machten wir die Beobachtung, dass der wasserhelle Urin eines an der schweren Form des Diabetes leidenden Patienten mit reiner (chlorfreier) Salzsäure versetzt, sich schön rosaroth färbte. Diese bis jetzt nicht beachtete Erscheinung regte uns zur genaueren Untersuchung derselben an, und ist es auch uns nicht gelungen, die farbige Materie in chemisch reinem Zustande darzustellen und zu analysiren, so haben wir doch ihre wichtigsten Eigenschaften kennen gelernt, wodurch es leicht gemacht ist, gegebenen Falls ihr Vorkommen nachzuweisen.

Diesen Farbstoff, den wir Uroroseïn nennen wollen, 